ORIGINAL ARTICLE

\title{
Outpatient rehabilitative treatment of chronic fatigue syndrome (CFS/ME)
}

\author{
R Viner, A Gregorowski, C Wine, M Bladen, D Fisher, M Miller, S El Neil
}

Arch Dis Child 2004;89:615-619. doi: 10.1136/adc.2003.035154

See end of article for authors' affiliations

Correspondence to:

Dr R Viner, Great Ormond

Street Hospital for

Children, Great Ormond

Street, London WCIN

3JH, UK;

R.Viner@ich.ucl.ac.uk

Accepted

23 November 2003
Aims: To assess the outcome of outpatient multidisciplinary rehabilitative treatment (graded activities/ exercise programme, family sessions, and supportive care) compared with supportive care alone for children and adolescents with chronic fatigue syndrome (CFS/ME).

Methods: Fifty six young people (aged 9-17 years) with CFS/ME by standard criteria were followed up for 3-24 months. All subjects received supportive care. Families additionally opted to either enter the rehabilitation programme (supportive care plus graded activities/exercise programme and family sessions) or have no additional treatment.

Results: Twenty two (39\%) subjects had supportive care alone and $26(46 \%)$ entered the programme. Treatment groups were comparable at baseline in terms of age, severity and duration of illness, Wellness score, and school attendance. At end of follow up, those in the programme group had significantly higher Wellness score and school attendance than those having supportive care alone. The programme significantly reduced the overall severity of illness: after the programme, $43 \%$ had complete resolution of CFS/ME compared to only $4.5 \%$ of those having supportive care alone. The presence of depressed mood and family beliefs about the aetiology of CFS/ME were not significantly associated with outcomes.

Conclusions: Outpatient rehabilitative treatment offers significant potential to improve the prognosis of CFS/ME in childhood and adolescence.
C hronic fatigue syndrome (CFS), also known as myalgic encephalomyelitis (ME), is characterised by medically unexplained severe physical and mental fatigue resulting in significant functional impairment that persists for more than 3-6 months. The management of CFS/ME in children and adolescents presents a common clinical challenge. Yet there is very little evidence to guide treatment of such children, ${ }^{1}$ particularly in an environment where there may be conflicting opinions regarding management of fatigue states. ${ }^{2}$ The recent UK Chief Medical Officer's Working Group on CFS/ME called for efforts to increase the evidence base for management of CFS/ME in young people. ${ }^{1}$ The need for effective interventions is shown by recent data suggesting that $63 \%$ of children and adolescents with CFS/ME treated with medical and supportive care had significant symptoms persisting 13 years after diagnosis. $^{3}$

No randomised controlled trials of CFS/ME in childhood and adolescence have been published. In adults, evidence from randomised trials has supported the effectiveness of cognitive behavioural therapy $(\mathrm{CBT})^{4}$ and graded exercise programmes. ${ }^{5}$ The application of CBT principles within a family setting for young people with CFS/ME is proving promising, ${ }^{6}$ but there have been no published trials of graded exercise programmes in adolescents.

A number of reports suggest that the most appropriate treatment for CFS/ME in children and adolescents is multidisciplinary rehabilitative treatment, with an emphasis on increasing activities alongside symptom management and addressing psychological issues through systemic (family) approaches. ${ }^{17-13}$ This approach is based on the heterogeneous and subjective nature of CFS/ME and a recognition that the syndrome may be best understood as a chronic state of low physical and emotional functioning where biological causal factors may no longer be operative but illness is maintained by physical deconditioning, sleep disturbance, and psychosocial factors. ${ }^{814} 15$
While rehabilitative approaches have been reported to have potential in the inpatient setting, ${ }^{15}$ no reports have been published on the outcome of multidisciplinary rehabilitation in an outpatient setting. Little evidence also exists concerning the benefits of alternative approaches to the management of CFS/ME, including non-interventionist treatment based on rest, pacing, and supportive care. ${ }^{12} 1617$ Despite sometimes contentious debate, there is little available evidence to favour the use of either rehabilitative or noninterventionist approaches in the management of young people with CFS/ME.

We used a non-randomised design to compare the effectiveness of an outpatient multidisciplinary rehabilitation programme with that of a non-interventionist supportive approach, using prospectively collected clinical data on young people with CFS/ME.

\section{METHODS}

Data were collected prospectively on all young people with CFS/ME seen by the joint Great Ormond Street Hospital and University College London Hospitals Adolescent Medicine Service between June 1998 and December 2002. Subjects were drawn from all parts of southeast England and were referred either by a local paediatrician for specialist management or by their general practitioner after having seen local paediatric services.

Eligible subjects were those who met the CDC diagnostic criteria for $\mathrm{CFS}^{18}$ modified for use with children and adolescents by using a three month duration of fatigue. ${ }^{19}$ We excluded subjects with treatable medical causes of fatigue, those on drugs known to cause fatigue, and those in whom somatoform disorder or school refusal was considered to be the diagnosis rather than CFS/ME.

\section{Treatment}

All subjects underwent combined outpatient medical and nursing assessment within 3-5 months of referral. All 
subjects who desired follow up received supportive care, including regular three monthly clinic medical review plus regular specialist nurse telephone support providing practical advice on self pacing, symptom control, and school reintegration. In addition, families were offered entry to a rehabilitation programme (involving a graded activities/ exercise programme and family work) or, for those with significantly depressed mood, the use of selective serotonin reuptake inhibitor (SSRI) medications. Those taking SSRI are not included in this analysis. Management plans were negotiated jointly between families and the clinical team, taking into account the beliefs of the family regarding the aetiology of the illness. While all families understood that the clinical team recommended a rehabilitative approach, they were assured that the team would support non-interventionist treatment if there were no child protection concerns. Ethical approval was not obtained for this study as we were comparing different forms of current management of CFS/ $\mathrm{ME}$ in young people.

\section{Rehabilitation programme}

Family sessions using a mix of structural, strategic, and systemic approaches were delivered by the nurse specialist together with either a social worker or nurse counsellor. Sessions focused on practical management issues, such as the active management of school reintegration, re-establishment of sleep routines, help with social care issues, and helping the family re-establish their pre-morbid functioning. The sessions also addressed more systemic issues such as how the CFS/ME affected each family member but specifically did not seek to identify "causes" or define "problems" in the families. Families were seen for an hour fortnightly to every few months depending on need. The median number of sessions was 6 (range 3-15).

In parallel with the family sessions, a graded activity and exercise programme was supervised by the team physiotherapist. The programme was based on principles of pacing and grading, initially focusing on daily activities with exercise introduced when appropriate. Detailed explanations to the families stressed the graded and negotiated nature of the programme, and stressed the importance of establishing a daily sustainable level of activity. Sessions occurred on the same day as family sessions, with telephone support between appointments. The ownership of the programme by the young person was emphasised, with activity and exercise choices made in negotiation with the physiotherapist.

\section{Outcomes}

Clinical data and treatment received were prospectively recorded at each clinic visit. A full clinical history and examination was undertaken at assessment. Families were asked about their beliefs concerning the origin of their child's illness and whether they believed that there was a psychological element to the illness. We aimed to follow subjects routinely every three months in clinic regardless of the severity of illness. Two primary outcome measures were recorded at each visit:

- Global Wellness score: The self rated single item global health Wellness score provides a subjective assessment of overall physical and mental wellbeing and has previously been used as a measure of overall health and quality of life in randomised treatment studies in adult CFS/ME. ${ }^{20}{ }^{21}$ Young people were asked in a standardised fashion to provide an average score for the previous month on a scale between 100 (the best they could imagine ever feeling) and 0 (the worst they could imagine feeling or feeling like dying).

- School attendance: Average school attendance in the previous three months was used as a marker of a young person's functional status and ability to participate in normal life. ${ }^{62223}$ Data from families were verified by regular telephone contact with the school or college. For those in colleges where attendance was only required for subjects taken, the percentage of required weekly contact was calculated.

Differences between treatment groups were assessed in three ways. (A) Firstly, group mean or median Wellness and school attendance at each time point during follow up were assessed. (B) Secondly, the best Wellness and school scores in the two clinic visits before the end of follow up were identified for each subject. As this was a clinical study of ongoing treatment, patients were at different points in their follow up when the study concluded in December 2002. End of follow up was defined as either December 2002 (if still under follow up) or when a subject was discharged from clinic. (C) Thirdly, Wellness and school attendance were combined to produce an estimate of disease severity at assessment and at the end of follow up.

Severity was defined as follows: (1) resolution of CFS: subjects with both a Wellness score of $\geqslant 90$ and school attendance of $\geqslant 95 \%$; (2) minimal CFS: subjects with both a Wellness score of $\geqslant 75$ and school attendance $\geqslant 75 \%$; (3) moderate CFS: those with either or both scores $\geqslant 50$ but $<75$; (4) severe CFS: those with either or both of Wellness score and school attendance $<50$. Where Wellness and school attendance were in different severity categories, subjects were assigned to the lower category.

\section{Analysis}

Treatment group differences were assessed using $t$ tests for normally distributed data and the Mann-Whitney U test for non-normally distributed data. The effects of treatment and personal factors on CFS/ME severity were assessed using $\chi^{2}$ tests.

\section{RESULTS}

Eighty five young people 9-17 years of age were referred for assessment for possible CFS/ME. Seventy nine (93\%) met the criteria for CFS. Seventy eight subjects $(92 \%)$ are included in this analysis; one subject ( $1 \%$ ) was excluded because of child protection concerns which made the clinical team unwilling to support the treatment desired by the family.

Table 1 shows details of the subjects at assessment. There were no significant differences between males and females on age at assessment, age of onset, duration of illness, or mean Wellness or school attendance at baseline. Severity scores could be assigned to 76 subjects (97\%), of whom $62 \%$ had severe CFS, 29\% moderate CFS/ME, and 9\% only minimal CFS. Families were evenly split regarding the possibility of a psychological element to the aetiology of their child's symptoms, with $51 \%$ accepting that this was a possibility but $49 \%$ reporting that they believed that their child's illness was entirely organic. A depressed mood was noted in $33(42 \%)$ but no subjects were considered by the team to have depression as their sole diagnosis.

\section{Treatment}

Data was available on outcome of treatment in 56 (72\%; 21 males, 35 females). The remainder were too early in treatment to have follow up data (12 (15\%), or had only been seen once and continued to be managed locally by the referrer $(10(13 \%))$. Follow up data were available on all 56 subjects at 6 months, 32 at 12 months, 24 at 18 months, and 17 at 24 months.

Twenty two subjects (39\%) had supportive care only and 26 (46\%) entered the programme. The remaining eight subjects (15\%) were prescribed SSRI either with supportive care or the 


\begin{tabular}{ll} 
Table 1 & Patient characteristics at assessment $(\mathrm{n}=78)$ \\
\hline Age (years), mean (SE) & $14.2(0.2)$ \\
Range & $9-17$ years \\
Sex, $n(\%)$ & $29(37)$ \\
Male & $49(63)$ \\
Female & $27.1(2.1)$ \\
Duration (months), mean (SE) & $12.2(0.2)$ \\
Age of onset (years), mean (SE) & $10.3(2.1)$ \\
Duration of follow up (months), mean (SE) & $52.0(2.5)$ \\
Wellness score, mean (SE) & $35.1(4.2)$ \\
School attendance, mean (SE) & $47(62)$ \\
Severity of CFS, n (\%) & $22(29)$ \\
Severe & $7(9)$ \\
Moderate & \\
Minimal &
\end{tabular}

programme and are not included in analysis. There were no significant differences between groups in terms of age, age at onset of CFS/ME, duration of illness, or baseline Wellness and school attendance (see table 2).

\section{Outcomes}

Median Wellness scores during follow up are shown in fig 1 and at end of follow up in table 2. Those in the programme had higher median Wellness scores from six months after beginning treatment, with this difference reaching significance at six months $(\mathrm{p}<0.01)$ and nine months $(\mathrm{p}<0.05)$. The programme resulted in a significantly higher median Wellness at end of follow up $(p=0.002)$ and a mean improvement in Wellness of $71 \%$ between baseline and end of follow up, over twice the improvement seen in the supportive care group $(p=0.05)$.

Mean school attendance during follow up is shown in fig 2 . Those in the programme had higher school attendance from nine months after beginning treatment, with this difference reaching significance at 12 months $(p=0.02)$. Those in the programme also had significantly higher median school attendance at end of follow up $(p=0.02)$ and a fivefold higher percentage improvement in attendance between baseline and end of follow up $(\mathrm{p}=0.01)$.

The impact of the programme on the severity of CFS/ME is shown in fig 3. At baseline there were comparable proportions in the different severity categories. Those in the programme group had significantly less severe CFS/ME at the end of follow up $\left(\chi^{2}=16.3, p<0.01\right)$, with $43 \%$ in the resolved category compared to only $4.5 \%$ of those having supportive treatment alone. Of the 17 subjects in the severe

Table 2 Wellness and school scores at assessment and end of follow up by treatment group

\begin{tabular}{lll}
\hline & Supportive care & Programme \\
\hline $\mathrm{n}(\%)$ & $22(39)$ & $26(46)$ \\
$\mathrm{Sex}, \mathrm{n}(\%)$ female & $13(59)$ & $15(58)$ \\
Age at assessment, mean (SE) & $14.4(0.4)$ & $13.9(0.3)$ \\
Age at onset, mean (SE) & $12.5(0.5)$ & $11.8(0.4)$ \\
$\begin{array}{l}\text { Duration (months), mean (SE) } \\
\text { Wellness score (median) }\end{array}$ & $28.1(4.0)$ & $25.7(4.8)$ \\
$\quad$ Assessment & 50 & 50 \\
$\quad$ End follow up & 67 & $85^{* *}$ \\
$\begin{array}{l}\text { Change in Wellness score, } \\
\text { mean \% (SE) }\end{array}$ & $31(11)$ & $71(19)^{*}$ \\
$\begin{array}{l}\text { School score (median) } \\
\quad \begin{array}{l}\text { Assessment } \\
\text { End follow up }\end{array}\end{array}$ & 40 & 20 \\
$\begin{array}{l}\text { Change in school score, } \\
\text { mean \% (SE) }\end{array}$ & 40 & $90^{*}$ \\
\hline
\end{tabular}

Difference between programme and supportive care: * $p<0.05$; ${ }^{* *} p<0.01$

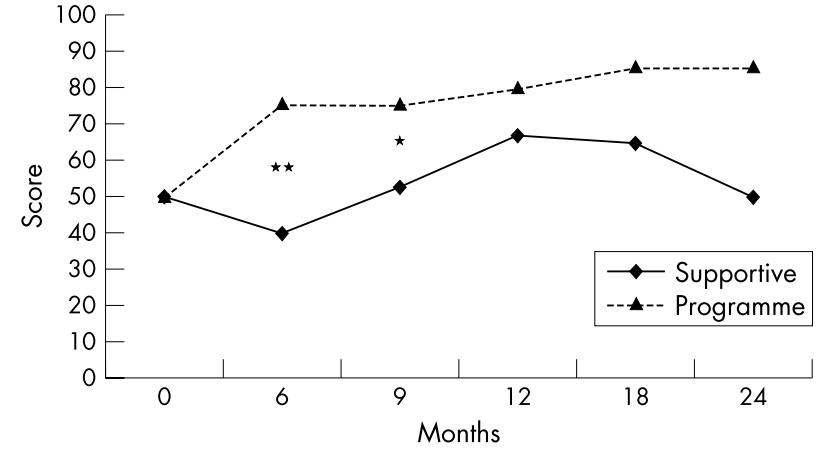

Figure 1 Median Wellness score in programme and supportive care groups during follow up. ${ }^{*} \mathrm{p}<0.05,{ }^{* *} \mathrm{p}<0.01$.

category who entered the programme, at the end of follow up six $(35 \%)$ were in the resolved category, three $(18 \%)$ in minimal, three (18\%) in moderate, and only $23.8 \%$ continued to have severe CFS/ME. By comparison, $63.6 \%$ of those with severe CFS/ME who had supportive care remained in the severe category.

No major individual factors were associated with response to treatment; however, improvement in Wellness score in the whole group was correlated with older age at onset $(r=0.32$, $\mathrm{p}<0.05)$ and shorter duration of illness $(r=-0.47$, $\mathrm{p}<0.001$ ), irrespective of treatment. Depressed mood or family beliefs about the aetiology of CFS/ME were not significantly associated with entry into treatment group, clinical outcomes, or response to treatment. Notably, the proportions of families who did not accept a psychological element to their child's illness were similar in both the programme (55\%) and supportive care (53\%) groups, as were the proportion of those with depressed mood at assessment (33\% of those in the programme compared with $36 \%$ of those having supportive care).

\section{DISCUSSION}

We obtained strongly positive outcomes from multidisciplinary outpatient rehabilitative therapy in young people with CFS/ME. Using prospectively collected data and a priori definitions of response to treatment, we found that a rehabilitative programme combining family work with an activities and exercise programme significantly improved subjective and functional outcomes compared with supportive treatment alone.

This is the first published report on outpatient rehabilitative treatment of CFS/ME and the first to compare rehabilitation with non-interventionist approaches. A previous uncontrolled study suggested that inpatient rehabilitative

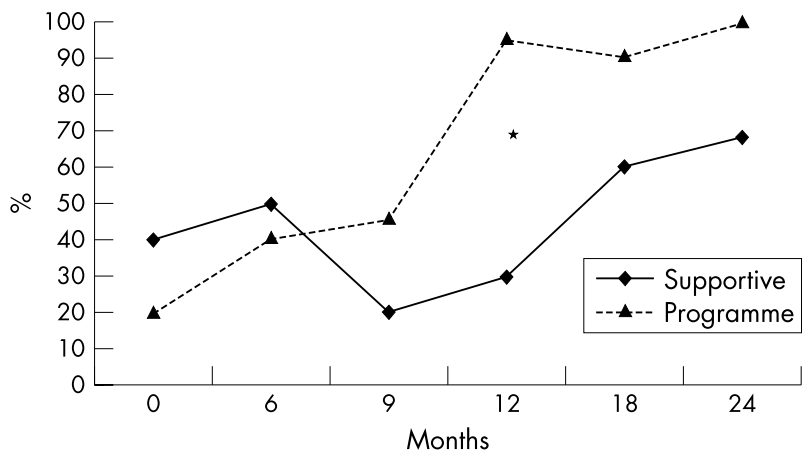

Figure 2 School attendance in the programme and supportive care groups during follow up. ${ }^{*} p<0.05$. 

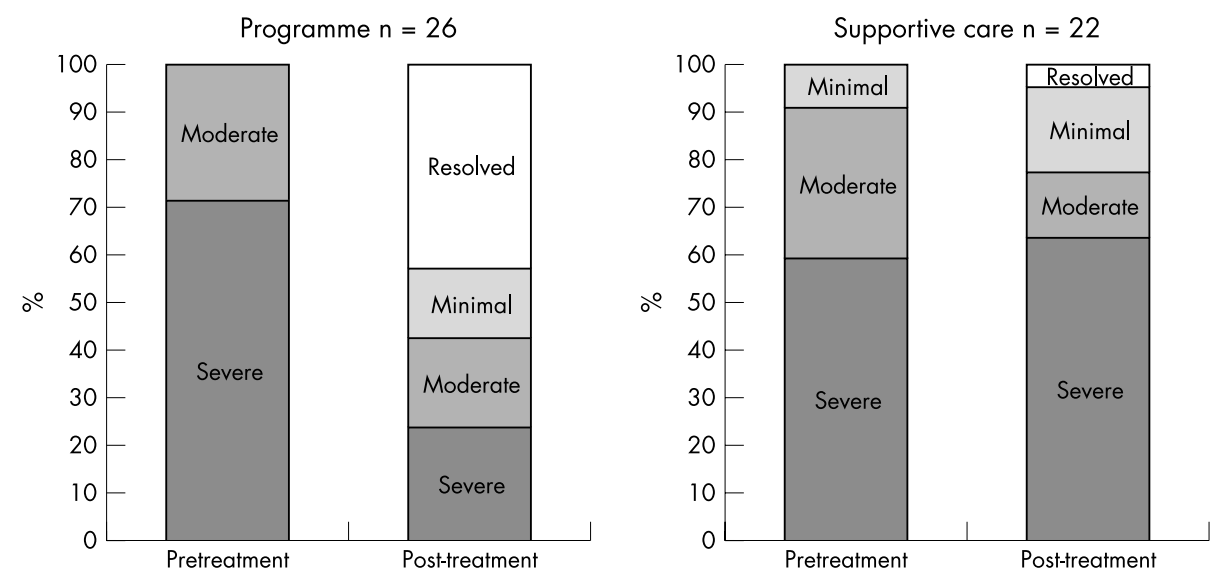

Figure 3 CFS severity after treatment in the programme compared with supportive care.

treatment can be effective in over half the subjects. ${ }^{15}$ Our data suggest that similar or better results can be obtained by outpatient management at potentially much reduced cost.

It is important to note that there were some clinical improvements in those having only supportive care, suggesting that gradual improvements and even complete resolution are possible without rehabilitative treatment in some young people with CFS. However, these gains were small and infrequent when compared with the results of rehabilitative treatment. It is possible that some of the improvements seen in those having supportive treatment alone are due to complementary treatments undertaken outside our service.

We were disappointed to find no significant indicators of response to treatment. Family beliefs about the possibility of a psychological element to the aetiology of CFS/ME and depressed mood at assessment did not influence entry into treatment groups and were not predictive of clinical outcome. The only non-treatment factor significantly associated with improvement was a shorter duration of illness, which may support the need for early intervention.

There are many limitations to this data. Firstly treatment groups were not randomised, thus selection bias may have significantly inflated treatment effects. Subjects who were more motivated to change are potentially more likely to have entered the programme. Secondly, subjects and families knew that supportive care was not the recommended treatment option for our clinic. Thirdly, intensity effects may also have operated; although the clinical team aimed to treat all patients to a similar intensity, those who actively engaged in our recommended treatment options may have received more intensive input. Fourthly, our clinic population may over-represent the more severe and chronic end of the CFS/ME spectrum, which means that these findings are not necessarily applicable to CFS/ME in the general population. However, a recent study suggests that the profile of patients seen in general practice is similar to that described from hospital clinics. ${ }^{24}$ Fifthly, we were not blinded to whether patients were on the programme or not. However, the two outcomes used were both generated by subjects themselves.

Despite these points, we believe that these findings strongly support the effectiveness of multidisciplinary rehabilitation over supportive care alone. Randomised treatment trials can be extremely difficult to undertake in complex subjective illnesses where differing health belief systems operate. While we could not negate the selection bias inherent in allowing families to choose treatment options, we note that the programme and supportive care alone groups were similar in terms of factors that may be a particular source of bias, containing nearly identical proportions of those with depressed mood and those who saw CFS/ $\mathrm{ME}$ as a purely organic illness. The programme and supportive care groups contained similar proportions of those with severe CFS, whereas none of the minimal CFS/ME cases opted for the programme, suggesting that bias from severity of illness may have operated to reduce rather than inflate the effects of the programme. Additionally, prospective collection of data and a priori identification of outcomes of interest and definitions of response serve to reduce observer bias.

The characteristics of our patient group at assessment were very similar to those noted by previous studies of CFS/ME in adolescence. ${ }^{32324}$ We found that just over $40 \%$ had depressed mood on clinical assessment, which accords well with previous findings that around half have psychiatric disorders, predominantly anxiety and depression. ${ }^{25}$

Our programme was undertaken within an adolescent paediatric medical setting in conjunction with mental health professionals. Given the frequent reluctance by CFS/ME subjects and their families to engage with mental health treatment, we suggest that basing joint paediatric-mental health working within the medical setting provides the most useful way to help families engage with rehabilitative treatment. We speculate that a potential key element of our success was that the role of the nurse specialist covered both the medical clinics and the family work, allowing families and young people to integrate medical and psychological approaches in a manner they might otherwise have found difficult.

\section{Conclusions}

We suggest that early outpatient rehabilitative treatment offers significant potential to improve the prognosis of CFS/ $\mathrm{ME}$ in childhood and adolescence and should be considered the preferred approach to the management of this illness. It would be desirable to confirm this using a randomised controlled study design.

\section{ACKNOWLEDGEMENTS}

This paper was written during a sabbatical period at the Centre for Adolescent Health, Royal Children's Hospital, Melbourne Australia. Russell Viner is supported by a MidCareer Award from the Health Foundation.

\section{Authors' affiliations}

R Viner, A Gregorowski, C Wine, M Bladen, D Fisher, M Miller,

S El Neil, Department of Adolescent Medicine, Great Ormond Street Hospital for Children and University College London Hospitals, London, UK

\section{REFERENCES}

1 CFS/ME Working Group. Report of the CFS/ME Working Group: Report to the Chief Medical Officer of an Independent Working Group. 2002. 
2 Speight ANP, Kumar P, Sabai J. Paediatric chronic fatigue syndrome in one health authority-epidemiology, spectrum of severity and natural history. Arch Dis Child 2000;82:A1.

3 Bell DS, Jordan KM, Robinson M. Thirteen year follow-up of children and adolescents with chronic fatigue syndrome. Pediatrics $2001 ; 105: 994-8$.

4 Prins JB, Bleijenberg G, Bazelmans E, et al. Cognitive behaviour therapy for chronic fatigue syndrome: a multicentre randomised trial. Lancet 2001:357:841-7.

5 NHS Centre for Reviews and Dissemination. Interventions for the management of CFS/ME. Effective Health Care 2002;7:1-12.

6 Chalder T, Tong J, Deary V. Family cognitive behaviour therapy for chronic fatigue syndrome: an uncontrolled study. Arch Dis Child 2002;86:95-7.

7 Richards J. Chronic fatigue syndrome in children and adolescents: a review article. Clin Child Psychol Psychiatr 2000;5:31-51.

8 Garralda ME, Rangel L. Annotation: Chronic fatigue syndrome in children and adolescents. J Child Psychol Psychiatry 2002;43:169-76.

9 Jordan KM, Landis DA, Downey MC, et al. Chronic fatigue syndrome in children and adolescents: a review. J Adolesc Health 1998;22:4-18.

10 Vereker M. Chronic fatigue syndrome: a joint paediatric-psychiatric approach. Arch Dis Child 1992;67:550-5.

11 Wright JB, Beverley DW. Chronic fatigue syndrome. Arch Dis Child 1998;79:368-74.

12 Pipe R. Family therapy in the treatment of chronic fatigue syndrome in adolescence. ACPP Review Newsletter 1995;17:9-16.

13 Calvert $\mathbf{P}$, Jureidini J. Restrained rehabilitation: an approach to children and adolescents with unexplained signs and symptoms. Arch Dis Child 2003;88:399-402.
14 De Lorenzo F, Xiao H, Mukherjee M, et al. Chronic fatigue syndrome: physical and cardiovascular deconditioning. QJM 1998;91:475-81.

$15 \operatorname{Lim}$ A, Lubitz L. Chronic fatigue syndrome: successful outcome of an intensive inpatient programme. J Paediatr Child Health 2002;38:295-9.

16 Lapp CW. Management of CFS in children: a practising clinicians approach. Journal of Chronic Fatigue Syndrome 1997;3:59-76.

17 Franklin A. How I manage chronic fatigue syndrome. Arch Dis Child 1998;79:375-8.

18 Fukuda K, Straus SE, Hickie I, et al. The chronic fatigue syndrome: a comprehensive approach to its definition and study. Ann Intern Med 1994; 121:953-9

19 Royal Colleges of Physicians, Psychiatrists and General Practitioners. Chronic fatigue syndrome. Report of a joint working group of the Royal Colleges of Physicians, Psychiatrists and General Practitioners, 1997.

20 McKenzie R, O'Fallon A, Dale JK, et al. Low-dose hydrocortisone for treatment of chronic fatigue syndrome. JAMA 1998;280:1061-6.

21 Straus SE, Dale JK, Tobi M, et al. Acyclovir treatment of the chronic fatigue syndrome: lack of efficacy in a placebo-controlled trial. N Engl J Med 1988:319:1692-8

22 Rangel L, Garralda ME, Levin M, et al. The course of severe chronic fatigue syndrome in childhood. J R Soc Med 2000;93:129-34.

23 Krilov LR, Fisher M, Friedman SB, et al. Course and outcome of chronic fatigue in children and adolescents. Pediatrics 1998;102:360-6.

24 Patel MX, Smith DG, Chalder T, et al. Chronic fatigue syndrome in children: a cross sectional survey. Arch Dis Child 2003;88:894-8.

25 Garralda E, Rangel L, Levin M, et al. Psychiatric adjustment in adolescents with a history of chronic fatigue syndrome. J Am Acad Child Adolesc Psychiatry 1999:38:1515-21.

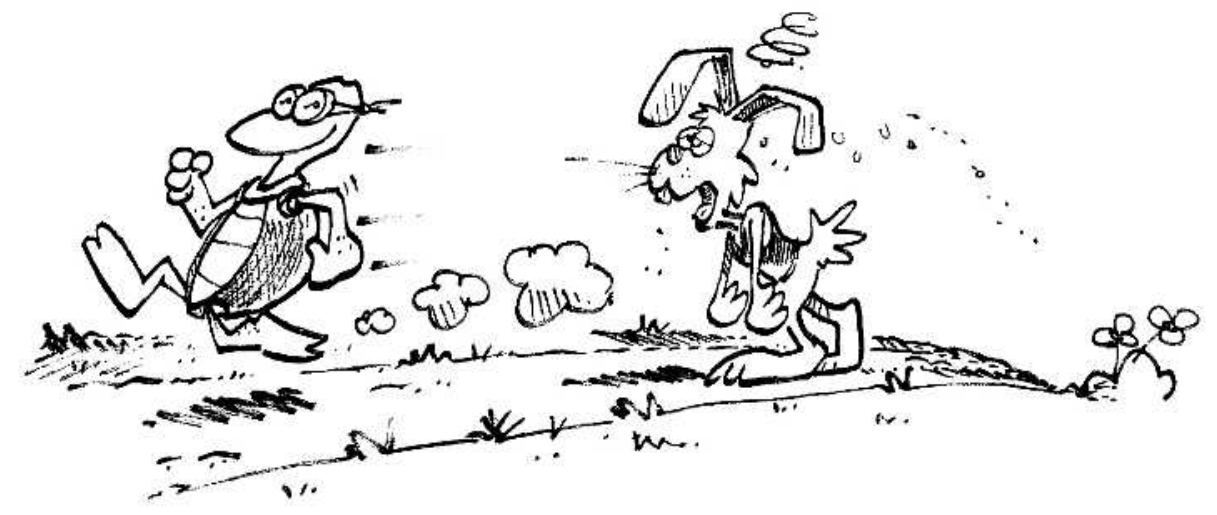

